\title{
INTERSTELLAR MOLECULAR HYDROGEN
}

\author{
PHILIP M. SOLOMON
}

Columbia University, New York, N.Y., U.S.A.

\begin{abstract}
The dominant photodissociation process for $\mathrm{H}_{2}$ under interstellar conditions is absorption in the lines of the ultraviolet Lyman bands at $912 \AA<\lambda<1108 \AA$ and re-emission into the continuum at $\lambda \sim 1600 \AA$. It is shown that $40 \%$ of all upward transitions in this band lead to emission into the continuum of the ground state and therefore dissociation of the molecule. The photodissociation rate is calculated and the theory is developed for the abundance of interstellar $\mathrm{H}_{2}$. It is shown that interstellar space will be divided into atomic and molecular regions in a manner analogous to the division between $\mathrm{H}_{\mathrm{II}}$ and $\mathrm{H} \mathrm{I}$ regions. The molecular regions will exist only where the rate of formation of $\mathrm{H}_{2}$ is sufficiently great to build up a self-shielding layer. This is expected in cold clouds with number densities greater than 100 atoms $\mathrm{cm}^{-3}$. Normal regions of interstellar space will have an extremely small abundance of molecular hydrogen.
\end{abstract}

